\title{
Adaptation of nonlinear systems through dynamic entropy estimation
}

\author{
Michał Żochowski and Rhonda Dzakpasu \\ Department of Physics and Biophysics Research Division, University of Michigan, Ann Arbor, \\ MI 48109, USA
}

Received 12 August 2003, in final form 20 November 2003

Published 28 January 2004

Online at stacks.iop.org/JPhysA/37/2223 (DOI: 10.1088/0305-4470/37/6/018)

\begin{abstract}
We show that adaptation and control of the target system can be achieved by linking the modification of its dynamical properties to the estimated difference of the distribution of the dynamical characteristics of the control and target signals. Subsequently the target system, which has initially different dynamical properties adjusts its dynamics via changes of its control parameters and synchronizes with the control one. The differences in the evolving probability distributions are evaluated through entropy estimation, causing the adaptation to be based solely on the statistical properties of the control and target signals without explicit knowledge of the underlying equations of the system.
\end{abstract}

PACS numbers: 05.45.-a, 05.45.Gg, 05.45.Xt, 05.65.+b

\section{Introduction}

The synchronization of two coupled identical nonlinear systems (having the same underlying equations and sets of control parameters) has been studied extensively [1-4]. However in many cases even though the dynamics of the systems are driven by the same underlying processes (i.e., they are described by the same sets of equations), the macroscopic properties of their trajectories will depend on the sets of control parameters. If these control parameters are different the simple coupling of trajectories between such systems cannot lead to synchronization since the properties of their orbits can be widely different (e.g., periodic versus chaotic) — the systems have effectively different dynamical properties. In this case, the target (driven) system must first adapt its dynamics to fit the driving pattern of the control system and then synchronize with the control system. There are many examples of systems in nature that rely on adaptation and synchronization for spatio-temporal pattern formation. Biological systems and the brain in particular are good examples [5]. Neurons exhibit complex patterns of spiking ranging from periodic to chaotic ones. Moreover, depending on the received signals the same cell can dramatically change its firing pattern (i.e., change from tonic firing to bursting and vice versa [6]) and synchronize with other neurons forming macroscopic spatio-temporal patterns. The resulting oscillations may appear as stable rhythms 
(alpha, beta, gamma) in the cortex [7-11] and/or as brief, stimulus evoked spatio-temporal patterns in different sensory modalities [12-17]. Formation of these patterns is thought to play a crucial role in information processing in the brain, as it is hypothesized that they underlie binding of features that are being computed by different cell populations but belong to the same information scene $[18,19]$.

The question approached here is whether and how two initially non-identical systems, i.e., having different sets of control parameters, and thus having different types of dynamics, can adapt and synchronize based solely on statistical and dynamical properties of their trajectories. We study the dynamics of two coupled systems: the control system that is unperturbed during its dynamics and the target system that is coupled to the control system. Information about the trajectory is transmitted through the single coupling and is integrated into the dynamics of the target on two different levels.

The adjustment of the target system with respect to the control system takes place over two different time scales. First, the target has to adjust its macroscopic properties so that the types of trajectories of the control and target systems are compatible. We refer to this process as adaptation. The adaptation happens on a relatively long time scale since it requires internal changes of the control parameter that define the type of the dynamic trajectory, and will depend on the slower changing macroscopic characteristics of the driving (control) signal. On the other hand, the synchronization of the trajectories already having similar properties takes place over a short time scale. This is accomplished by the direct coupling of iterates or coordinates to the target. These time scales are much shorter since the target is perturbed instantaneously by the trajectory of the control system. However, the two processes are intertwined as the synchronization cannot happen if the trajectories have different dynamical properties. On the other hand, the adaptation process stops when the two trajectories become identical due to their synchronization.

We show that monitoring of the relative differences of the distributions of dynamical properties of the control and target trajectories in conjunction with the direct coupling of the two systems may lead to successful adaptation and synchronization of the two systems. For it to be successful one has to define a measure that is able to follow the dynamical changes of the system during its adaptation. Invariants of attractors and specifically entropy estimation from time series is often used to characterize the state (or trajectory) of the studied system or to determine its dynamics [20-23]. However, to successfully estimate the properties of the signal one has to assume that the obtained time series are generated by a stationary process.

Here we assume that the adaptation is slow enough that it is possible to reliably estimate the dynamical characteristics of the system even though the macroscopic properties of the target trajectories are changing. To monitor these changes we use the evolving distributions characterizing the properties of the trajectory at a given time to calculate the entropy of the system. The differences between the entropies of the target and control are then used to drive the internal changes of the control parameter of the target system.

\section{Entropy estimation from distribution of trajectory characteristics}

The macroscopic properties of the trajectory of the system depend critically on the values of its control parameters. The system trajectory can change from chaotic to periodic due to small perturbation of this parameter. On the other hand, if the systems are driven by the same processes (described by the same equations) it may be possible to derive a correct set of control parameters that would allow the target system to mimic the behaviour of the control system. Such information may be useful for coding/decoding systems where the time series could be used to determine the properties of the coding system by the decoder. 
After the seminal work of Shannon [24], the entropy measurement is often used in information theory to obtain the statistical properties of the system and is directly linked to its dynamical properties. Here, we estimate the entropy from the time series obtained from the system trajectory to quantify its dynamical characteristics. The difference between the entropies of the target and control is used to adjust the value of the control parameter of the target so that the macroscopic properties of its trajectory match those of the control. In practical application the change of a control parameter should be viewed as an adjustment of the processes underlying the monitored system.

The time series is obtained by defining and monitoring events that will characterize well the dynamical trajectory of the system. During the evolution of the coupled system every registered event that is used to construct the distribution changes that distribution by a fixed amount. Specifically, if the $I$ th event occurs at time $t$, then

$$
P_{I}(t)=P_{I}(t-1)+\Delta P
$$

where $P_{I}(t)$ is the probability of the event $I$ happening at time $t$, and $\Delta P$ is the probability gain assigned to the group of events that the latest event belonged to. The resulting distribution is then renormalized so that $\sum_{I} P_{I}(t)=1$. Thus, the constructed distribution evolves with the changing properties of the system and is effectively skewed towards the newest events, whereas the older ones are forgotten exponentially. The rate of their decay depends on the $\Delta P$, so that if event $q$ did not happen during $n$ updates its probability at time $t$ declines to

$$
P_{q}(t)=\frac{1}{(1+\Delta P)^{n}} P_{q}(t-n) .
$$

The entropy is estimated simultaneously for the control and target systems from the changing probability distributions:

$$
S(t)=-\sum_{I} P_{I}(t) \ln P_{I}(t) .
$$

Figure 1 shows two examples of the distributions obtained from logistic maps using different values of the control parameters. The values of the control parameter $r$ were chosen in such a way that the distribution presented in figure $1(A)$ is obtained from a periodic regime of the logistic map whereas the distribution in figure $1(B)$ is obtained from the chaotic one. The entropy is calculated for both cases. The two distributions as well as entropy values are widely different indicating that the basic macroscopic characteristics of the trajectories can be captured and used to control the trajectory of the target.

We have plotted the difference in the entropies for different values of the control parameter for two uncoupled identical systems. Figure 2 presents the difference in entropy $\left(\Delta S=S_{1}-S_{2}\right)$ calculated from the distributions of the iterates of two uncoupled logistic maps (figure $2(A)$ ) and the distributions of the $x$-coordinates of the intersection between trajectories of uncoupled Rössler oscillators and the predefined Poincaré section (figure 2(B)), plotted as a function of the difference between the control parameters. In both cases, the entropy is essentially a monotonic function of the parameter difference, except for periodic windows that identify themselves as abrupt spikes in each plot. The proposed adaptation scheme relies on such monotonic dependence of $\Delta S$ on the parameter changes, since in the presented applications the sign of the derivative of the change in the control parameter of the target system is linked to the sign of the difference between the control and target entropies. One can generalize the search for the zeros of the expression $S_{1}-S_{2}$ in the parameter space and then the algorithm would not be limited to regions of the monotonic entropy dependence on the control parameter.

As will be shown in the examples below, the dynamical properties of the control and target trajectories can be estimated based on the distributions of different characteristics of 


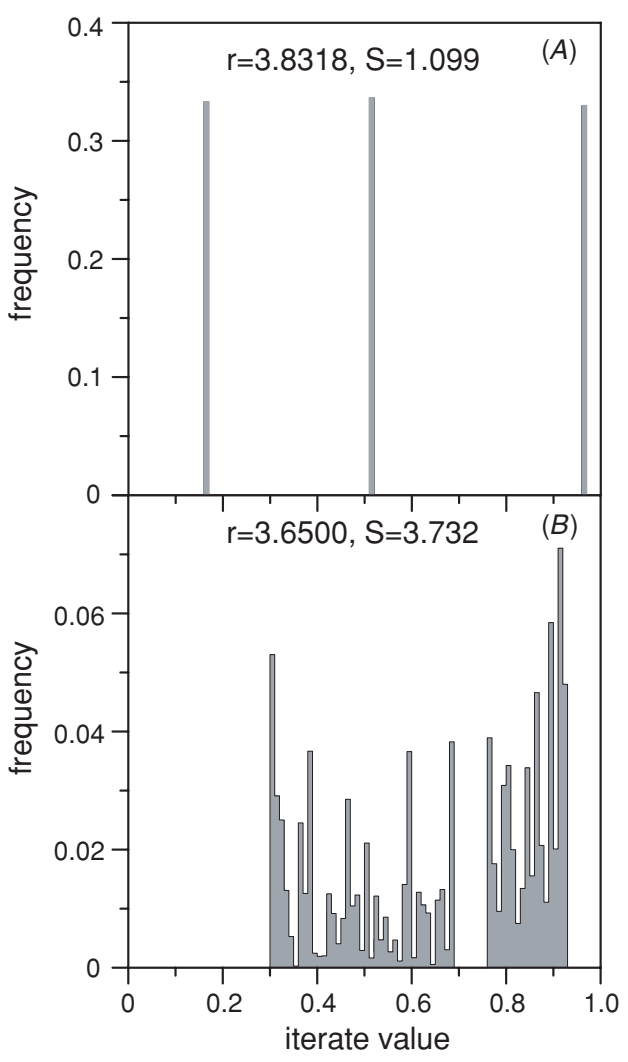

Figure 1. The distribution of the events obtained from equation (1) for different regimes of the logistic map. (A) Periodic regime obtained for the value of $r=3.83718$ (periodic window); (B) chaotic regime $(r=3.6500)$. The distribution was calculated for 100 bins and $\Delta P=0.01$.

the signal. Other characteristics of the signal to those presented below could also be used, for example the timings of the specific events (i.e. neuron emitting a spike).

\section{Adaptation and synchronization of two logistic maps}

We applied the entropy estimation to control trajectory of the target to a system of two coupled logistic maps. The equations of the coupled logistic maps are given by

$$
\begin{aligned}
& x_{1}(n+1)=f\left(x_{1}(n), r_{1}\right) \\
& x_{2}(n+1)=(1-\alpha) f\left(x_{2}(n), r_{2}(n)\right)+\alpha x_{1}(n+1)
\end{aligned}
$$

and

$$
f(x(n), r)=r x(n)(1-x(n))
$$

where $\alpha$ is a coupling constant, and $r_{1}$ and $r_{2}(n)$ are the control parameters of the control and target, respectively. While $r_{1}$ is constant throughout the dynamics, $r_{2}(n)$ is adjusted according to the equation

$$
\Delta r_{2}(n)=\beta\left(S_{1}(n)-S_{2}(n)\right) .
$$

The changes in the control parameter of the target depend on the sign and magnitude of the differences between the entropies of the two coupled systems, $\Delta S(n)$. The entropies in this 

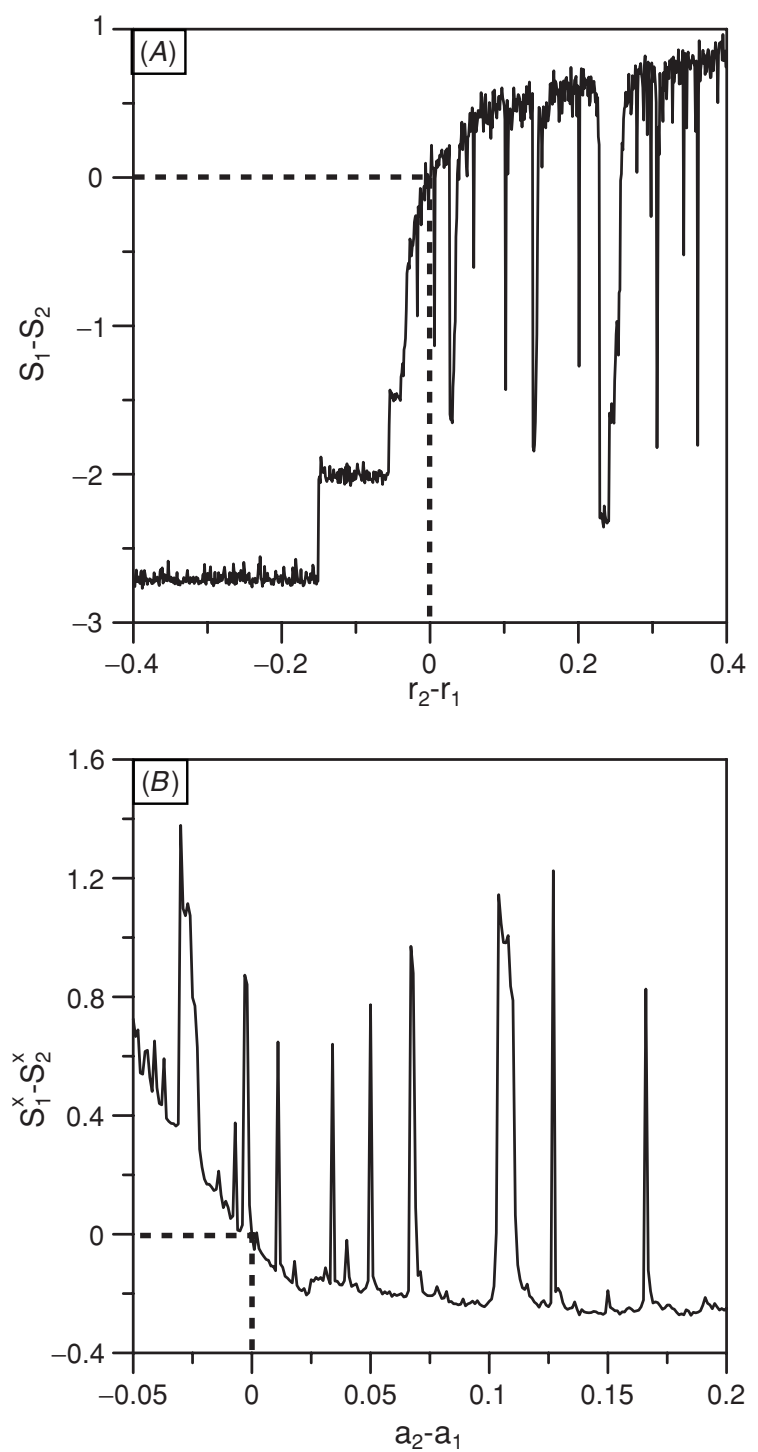

Figure 2. Entropy as a function of the difference in the control parameters: $(A)$ two uncoupled logistic maps (values of the control parameters are: $r_{1}=3.6, r_{2} \in[3.2,4]$ ) and $(B)$ two uncoupled Rössler oscillators (values of the control parameters are: $\left.a_{1}=0.15, a_{2} \in[0.1,0.35]\right)$. To estimate the entropy for the logistic maps we used the distributions of their iterate values, whereas for the Rössler oscillators we used the distribution of the $x$-coordinate of the trajectory points that intersected the Poincaré section given by the equation $y=x$.

case are estimated from the frequency distribution of the iterate values. The properties of this distribution depend on the value of the control parameter. As the two systems converge, the iterate values overlap and the $\left|S_{2}-S_{1}\right| \rightarrow 0$ and $\Delta r(n) \rightarrow 0$. The speed of the convergence will depend on the parameter $\beta$. However if the changes of parameter $\Delta r_{2}(n)$ are too fast in comparison with the changes of the entropy of the target the convergence will be reduced or never achieved. The control parameter of the target will overshoot the value of that of the 


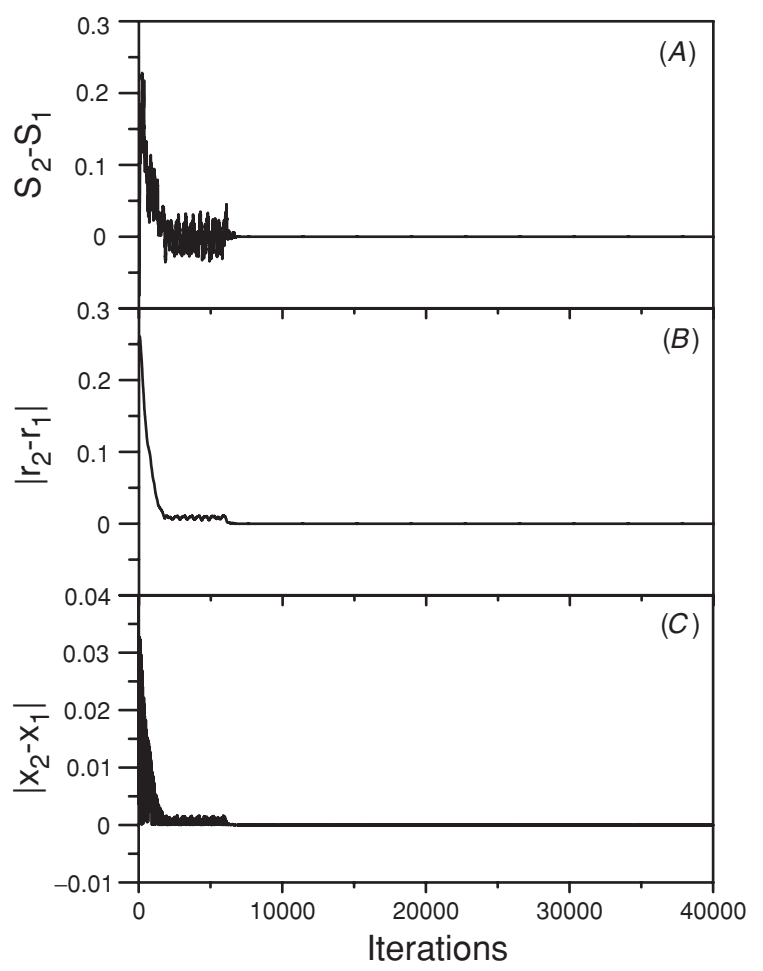

Figure 3. Adaptation and synchronization in a system of two coupled logistic maps: $(A)$ difference between estimated entropies of target and control systems; $(B)$ convergence of control parameters of the target and control maps; $(C)$ synchronization of their trajectories as a function of iterates. The target system rapidly adjusts its control parameter and the two systems synchronize. Simulations were performed for $r_{1}=3.65, r_{2}(0)=3.4, \alpha=0.5, \Delta P=0.01, \beta=0.02$.

control and start oscillating around it or diverge altogether. Thus the allowable magnitude of parameter $\beta$ will critically depend on the update rate of the probability distributions $(\Delta P)$.

The results of the simulations are presented in figure 3. Initially, $r_{1}=3.65, r_{2}(0)=3.4$ and the coupling constant $\alpha=0.5$. The initial values of the iterates are generated at random. Figure 3 shows the difference of entropies $\Delta S$ (figure 3(A)), difference of the parameters $\Delta r$ (figure $3(B)$ ), and difference of the iterates $\left|x_{2}-x_{1}\right|$ (figure $3(C)$ ) between the control and target maps. Initially the control parameters and the entropy are significantly different for the two systems. The $\Delta S(n)$ drives the changes of the control parameter of the target. As the $r_{2}(n) \rightarrow r_{1}$ the entropic difference tends to zero, so that the control parameter of the target eventually stabilizes to that of the control. At the same time $\left|x_{2}-x_{1}\right| \rightarrow 0$ as the two systems synchronize. In turn, since the two trajectories become synchronized the distributions become identical and $\Delta S$ will remain zero making the synchronized solution stable. As a result, even though the control and the target initially exhibit very different types of dynamics (target is periodic and control is chaotic) the target adapts and the two maps synchronize.

We studied the robustness of the applied control scheme in two different ways. First we investigated the convergence of the control and target systems and the subsequent synchronization for different values $r_{1}$ and $r_{2}$. Secondly, we investigated the synchronization and adaptation quality in the presence of noise. 


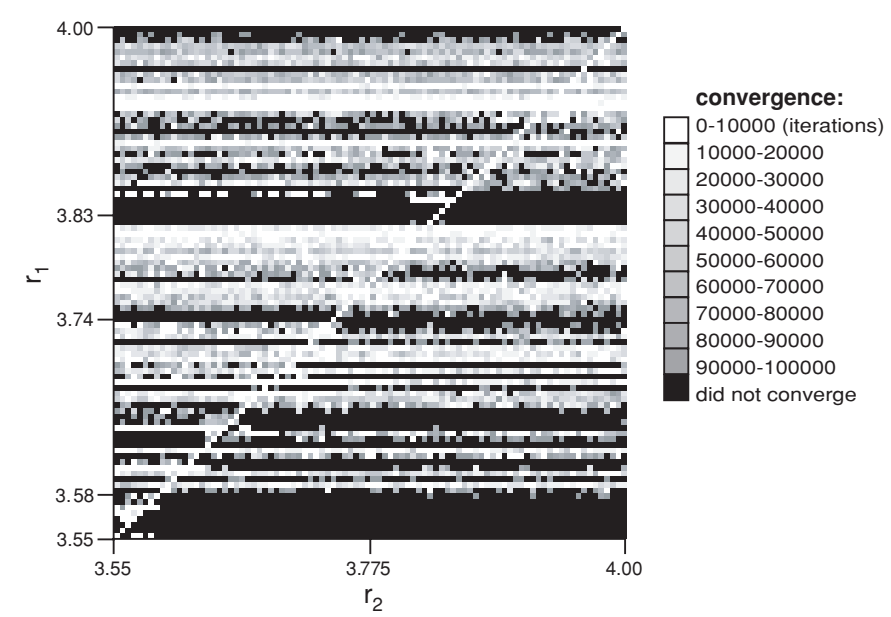

Figure 4. Convergence map for two coupled logistic maps. The average value of iterations at which adaptation and synchronization were achieved is converted to grey scale and plotted as a function of the value of the control parameter of the control unit $\left(r_{1}\right)$ and the initial value of the control parameter of the target unit $\left(r_{2}(0)\right)$.

Figure 4 is a grey scale map of the convergence and synchronization speeds of the coupled systems plotted as a function of the value of the control parameter of the control unit $\left(r_{1}\right)$ and the initial value of the target control parameter $\left(r_{2}(0)\right)$. The speed of convergence was determined by monitoring the average synchronization error $\left(\left|x_{1}(n)-x_{2}(n)\right|\right.$ in the case of logistic maps) and the average parameter convergence error, $\left|r_{1}-r_{2}(n)\right|$. The two quantities were averaged over 2500 iterations. If, simultaneously, both quantities were below the predefined values ( $E_{s} \leqslant 0.001$ for the synchronization error and $E_{r} \leqslant 0.005$ for the parameter convergence) the simulation was halted and the value of the last iteration was recorded. The simulation was repeated 10 times for every value of $r_{1}$ and $r_{2}$ and the iteration values were averaged. The average iteration values were then mapped onto the grey scale as shown in the legend next to the convergence map. A black square denotes a lack of convergence.

As expected, the convergence does not depend on the initial value of the control parameter of the target. The speed of convergence is similar for lower and higher values of the $r_{1}$. The regions depicting a lack of convergence overlap with the periodic windows of the logistic map. This is due to the fact that at these points the monotonicity of the entropy as a function of control parameter is broken. Also, the convergence is not achieved for the cases when the control unit is not chaotic. This could be explained by the fact that the value of the entropy will be the same for orbits of the same periodicity independent of the distributions of specific values of iterates in those orbits.

To study the effects of noise on the proposed control scheme we added a random variable to both systems. Thus, the equations now read

$$
\begin{aligned}
& x_{1}(n+1)=f\left(x_{1}, r_{1}\right)+A_{N} \xi_{1} \\
& x_{2}(n+1)=(1-\alpha) f\left(x_{2}, r_{2}\right)+\alpha x_{1}+A_{N} \xi_{2}
\end{aligned}
$$

where $A_{N} \in[0,0.05]$ is the noise amplitude and $\xi \in(-1,1)$ is a uniformly distributed random variable. We measured the mean divergence of the iterates of the logistic maps as well as the mean difference between the control parameter of the target and control units during the last 10000 iteration steps. The results are presented in figure 5. The $\left|r_{1}-r_{2}\right|$ is plotted in figure $5(A)$, whereas the synchronization error is plotted in figure $5(B)$. The black line in 


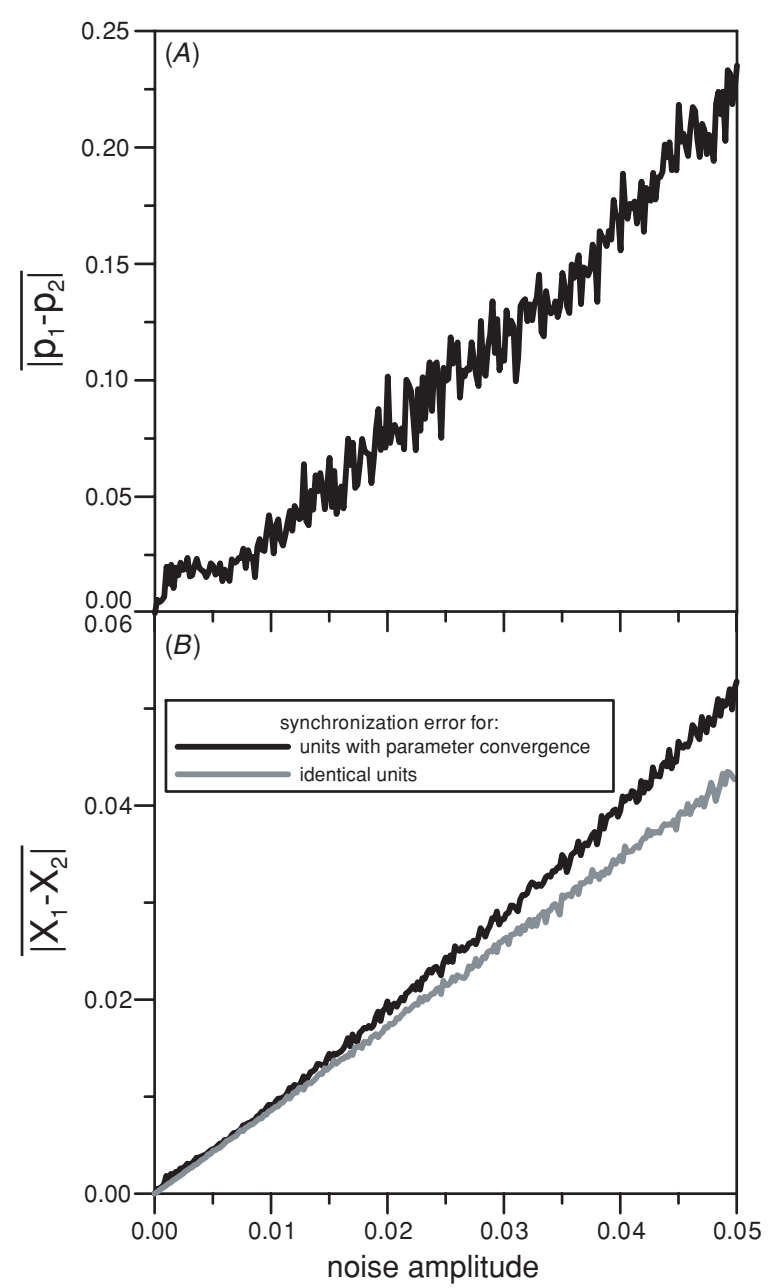

Figure 5. Synchronization and adaptation quality as a function of the noise amplitude for coupled logistic maps. (A) Plot of the convergence of the control parameters; $(B)$ the synchronization error. In $(B)$ the black line denotes the synchronization error of the two adapting maps $\left(r_{1}=3.65\right.$ and $\left.r_{2}(0)=3.5\right)$; the grey curve denotes the synchronization error of two identical maps $\left(r_{1}=r_{2}=3.65\right)$ in the presence of noise.

figure $5(B)$ represents the average difference between the iterates of the control and target units at the end of the adaptation process of the target (initially $r_{1}=3.65$ and $r_{2}(0)=3.5$ ). The grey line denotes the synchronization error for two identical coupled noisy logistic maps $\left(r_{1}=3.65\right.$ and $r_{2}=3.65$ are constant during the simulation). As would be expected the synchronization quality is reduced in the presence of noise. However, the synchronization error of the adapting systems is on average only $20 \%$ larger than that for two coupled identical units.

To achieve the reported adaptation and synchronization of the system we applied a large coupling constant $\alpha$. Since it has been reported [25] that synchronization of two logistic maps may take place even when their control parameters are different if a large coupling constant is used, we ran a control experiment in which the target did not adapt in order to check whether the observed synchronization is not an artefact of a large coupling. The results are plotted 


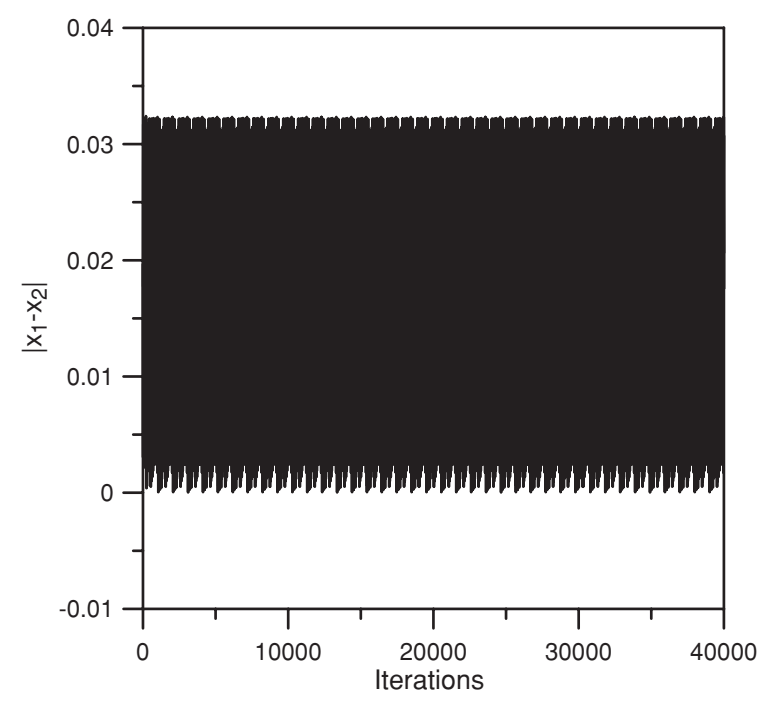

Figure 6. Lack of synchronization in a system of two coupled logistic maps with deactivated adaptation. The control parameters of control and target maps remain different throughout the simulation. The difference between the iterates of target and control maps is plotted as a function of iterates. Simulations were performed for $r_{1}=3.65, r_{2}(0)=3.4, \alpha=0.5, \Delta P=0.0, \beta=0.0$.

in figure 6. The target and the control systems remain desynchronized showing the importance of the adaptation in the process of synchronization of initially two non-identical systems.

\section{Adaptation and synchronization of two Rössler oscillators}

We applied the same procedure to control adaptation and synchronization of a system of two coupled Rössler oscillators. The differential equations of the coupled system are given by

$$
\dot{x}_{1}=-\left(z_{1}+y_{1}\right) \quad \dot{y}_{1}=x_{1}+a_{1} y_{1} \quad \dot{z}_{1}=b+\left(x_{1}-c\right) z_{1}
$$

for the control, and

$\dot{x}_{2}=-\left(z_{2}+y_{2}\right) \quad \dot{y}_{2}=x_{2}+a_{2}(t) y_{2}+\alpha\left(y_{1}-y_{2}\right) \quad \dot{z}_{2}=b+\left(x_{2}-c\right) z_{2}$

for the target oscillator. The two oscillators are coupled via their $y$-coordinate with $\alpha=0.3$. The parameters for the control system are $a_{1}=0.15, b=0.2, c=10.0$, making the control trajectory a chaotic one (the Liapunov exponents for this trajectory are: $\lambda_{1}=0.09, \lambda_{2}=$ $\left.0.00, \lambda_{3}=-9.80\right)$. Parameters $b$ and $c$ of the target have the same value as for the control system; $a_{2}(t)$ undergoes changes as the target system adapts its dynamics to match the control trajectory.

Additionally, we defined a Poincaré section (figure 7) $x=y$ and monitored the values of the $x$-coordinate at which the trajectories of the target and control systems intersect the plane. The entropy is estimated from the distribution of those points. Thus, every time the trajectory of the control or the target system intersects the Poincare section the respective distributions are updated according to equations (1) and (2) and the values of appropriate entropies are updated. As in (6) the difference between the entropies of both systems is used to update the parameter of the target:

$$
\Delta a_{2}(t)=\gamma\left(S_{2}(t)-S_{1}(t)\right) .
$$




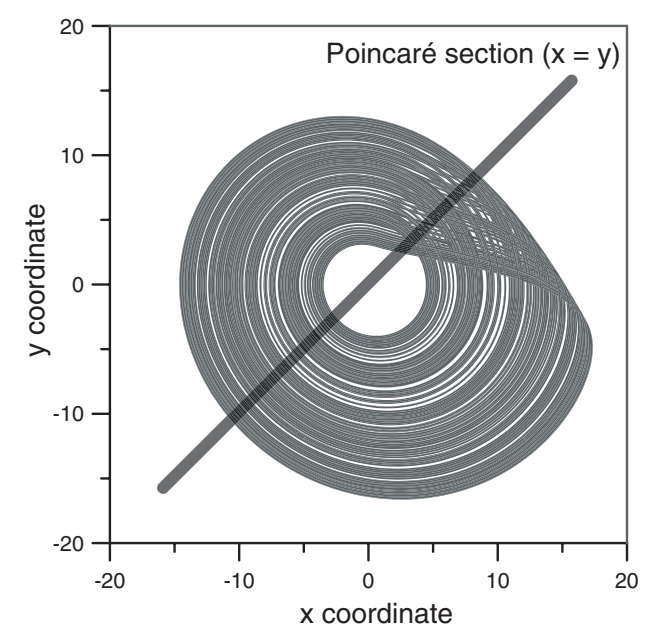

Figure 7. Trajectory of the Rössler oscillator and the Poincaré section used to estimate the distribution of the plane intersection coordinates. The entropies are estimated from the distribution of the $y$-coordinate of the intersection of the trajectory with the Poincare section.

Thus the control parameter of the target system $\left(a_{2}(t)\right)$ is updated by the same amount for every integration step until the trajectory of one of the oscillators intersects the Poincaré section. Then the distributions are updated and a new $\Delta a_{2}$ is calculated. The parameter $\gamma$ will determine the convergence speed. However, as before, its maximal value depends on the speed of the updates of the distributions $\Delta P$ that determine the values of the entropies.

Simulation results are shown in figure 8 . The initial value of the control parameter of the target system is $a_{2}(0)=0.3$; the initial trajectory points are randomly generated. Figure $8(A)$ shows the difference between the entropies of the two coupled oscillators. This difference is driving the convergence of the control parameters of the target and control (figure $8(B)$ ). Figure $8(C)$ shows the changes of the synchronization error,

$$
E(t)=\sqrt{\left(x_{2}-x_{1}\right)^{2}+\left(y_{2}-y_{1}\right)^{2}+\left(z_{2}-z_{1}\right)^{2}} .
$$

The synchronization error decreased by seven orders of magnitude throughout the simulation indicating convergence of the trajectories of the two oscillators.

We ran a control experiment when the adaptation was deactivated (i.e., $\gamma=0$ ), so that the difference between the control parameters of the target and control was constant throughout the simulation. The $E(t)$ is plotted for this case in figure 9. The trajectories of the target and control systems remain significantly different throughout the simulation, underscoring the role of the adaptation in the process of synchronization of the two trajectories.

As in the previous section we investigated the robustness of the convergence as a function of the control parameters for the target and control oscillators and, separately, in the presence of noise. Figure 10 depicts the convergence time for different values of the control parameters of the control and target oscillators. As with the logistic maps, the simulation was halted if the average convergence error of the control parameters and the average synchronization error were both less than $E_{s}=0.01$ and $E_{a}=0.001$. The average convergence time was then mapped onto a grey scale to create the convergence diagram. The simulations were performed for $\alpha=0.3$. The two coupled oscillators adapt and synchronize rapidly for most of the values of $a_{1}$, except for a few periodic windows. For $a_{1} \geqslant 0.27$ no convergence is obtained. This is due to the fact that the synchronized state for $\alpha=0.3$ is not stable for the higher values 


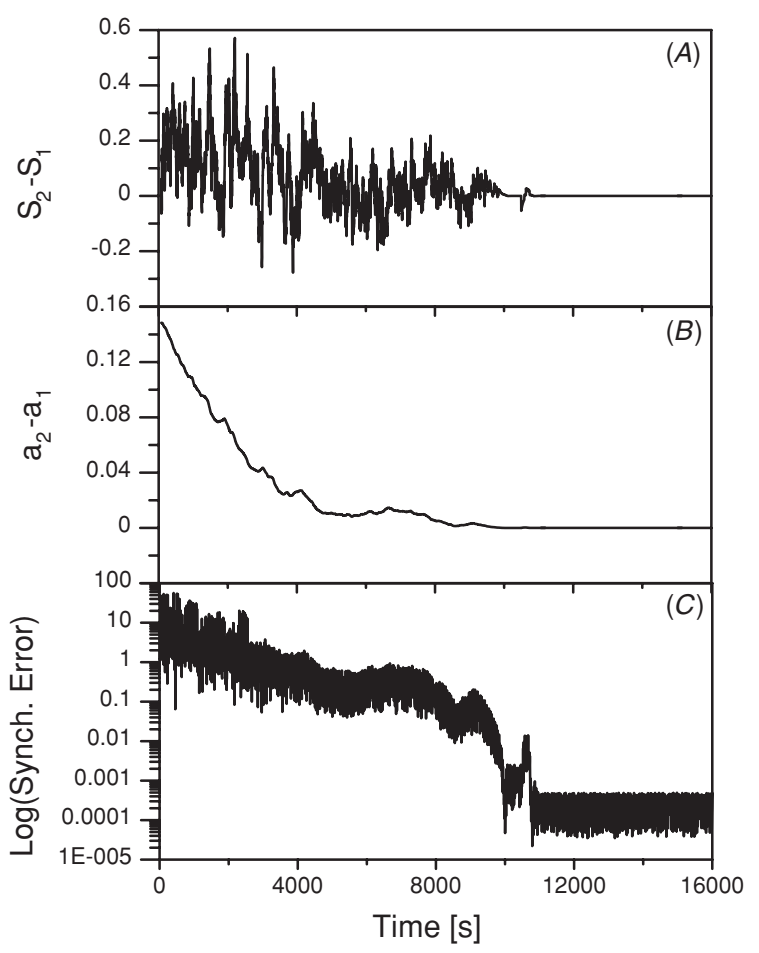

Figure 8. Adaptation and synchronization in a system of coupled Rössler oscillators: $(A)$ difference between entropies of target and control; $(B)$ convergence of control parameters $\left(a_{1}\right.$ and $\left.a_{2}(t)\right)$; $(C)$ synchronization error $E$. The two oscillators synchronize, reducing the synchronization error by seven orders of magnitude. Simulations were performed for $a_{1}=0.15, b=0.2, c=10.0, a_{2}(0)=$ $0.3, \alpha=0.3, \Delta P=0.05, \gamma=0.0004$.

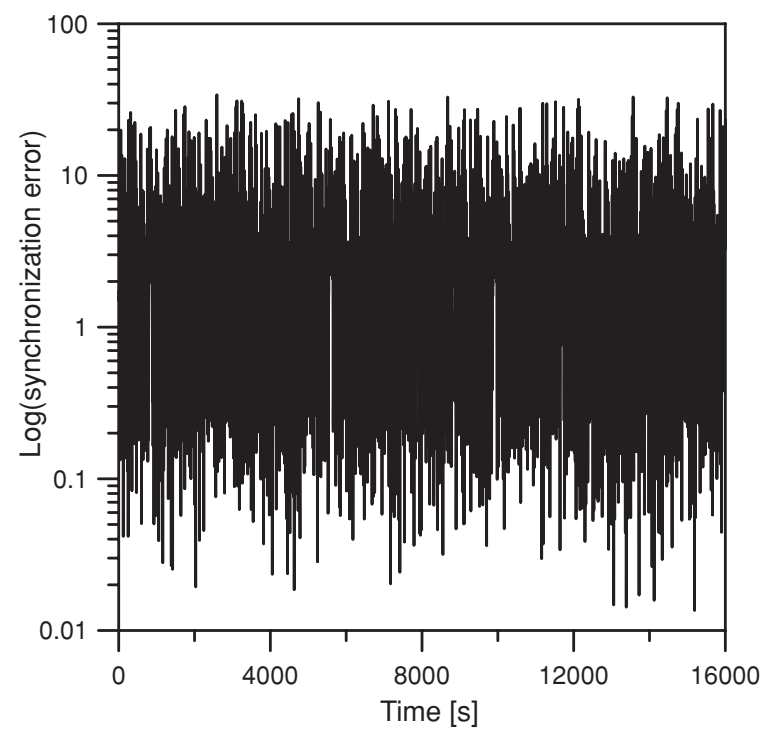

Figure 9. Lack of synchronization in a system of two coupled Rössler oscillators with deactivated adaptation. Synchronization error $E$ is plotted as a function of time. Simulations were performed for the same set of control parameters as in figure 8 . 


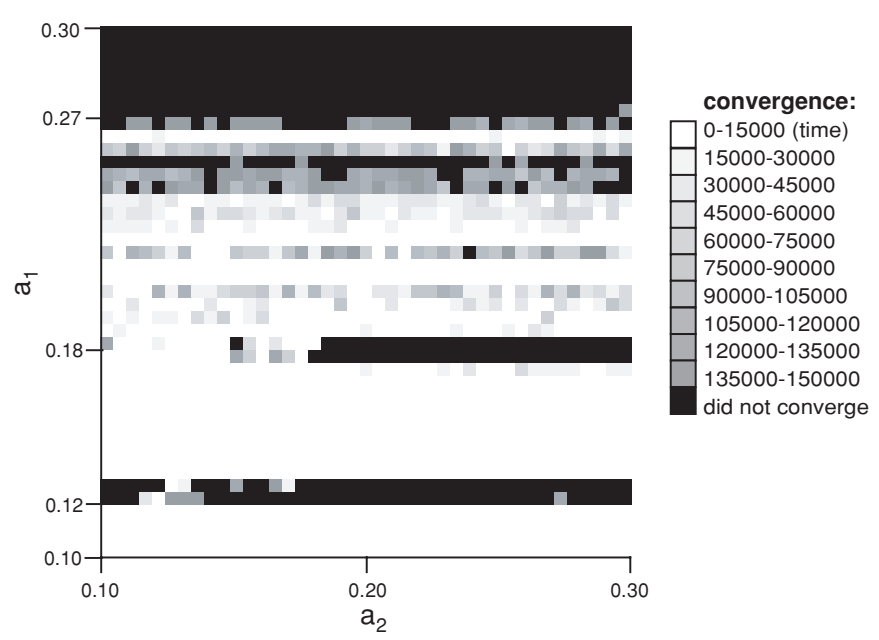

Figure 10. Convergence map for two coupled Rössler oscillators. The average value of the iterations in which adaptation and synchronization were achieved is mapped to a grey scale and plotted as a function of the control parameter of the control unit $\left(a_{1}\right)$ and the initial value of the control parameter of the target unit $\left(a_{2}(0)\right)$.

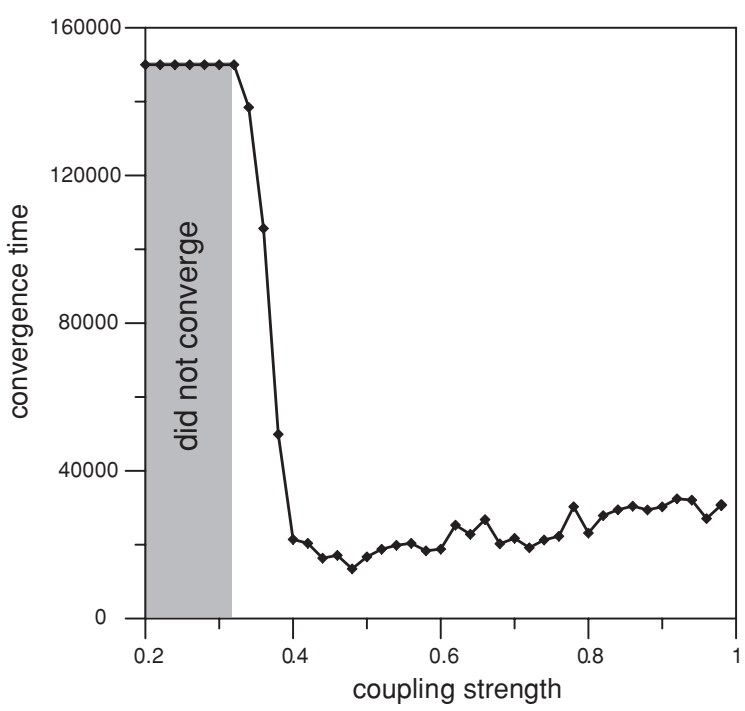

Figure 11. Time of convergence as a function of coupling strength for two Rössler oscillators. Initially $a_{1}=0.29$ and $a_{2}=0.10$. The convergence is not achieved for the value of coupling lower than $\alpha=0.34$.

of the control parameter $a_{1}$. Figure 11 shows the convergence time as a function of coupling strength $\alpha$. The value of the parameter of the control oscillator was set to $a_{1}=0.29$ and for the target oscillator, $a_{2}(0)=0.10$. Every point is an average of 20 simulation runs. There is no convergence when the coupling is below $\alpha=0.34$. Above this value the convergence time improves rapidly. We note that the lowest average time of convergence is achieved for $\alpha=0.4$. As the coupling is increased, the convergence time slowly increases. This may be due to the fact that as the coupling gets stronger the distributions used to calculate the entropy for the target unit are more skewed in the direction of the control one. 


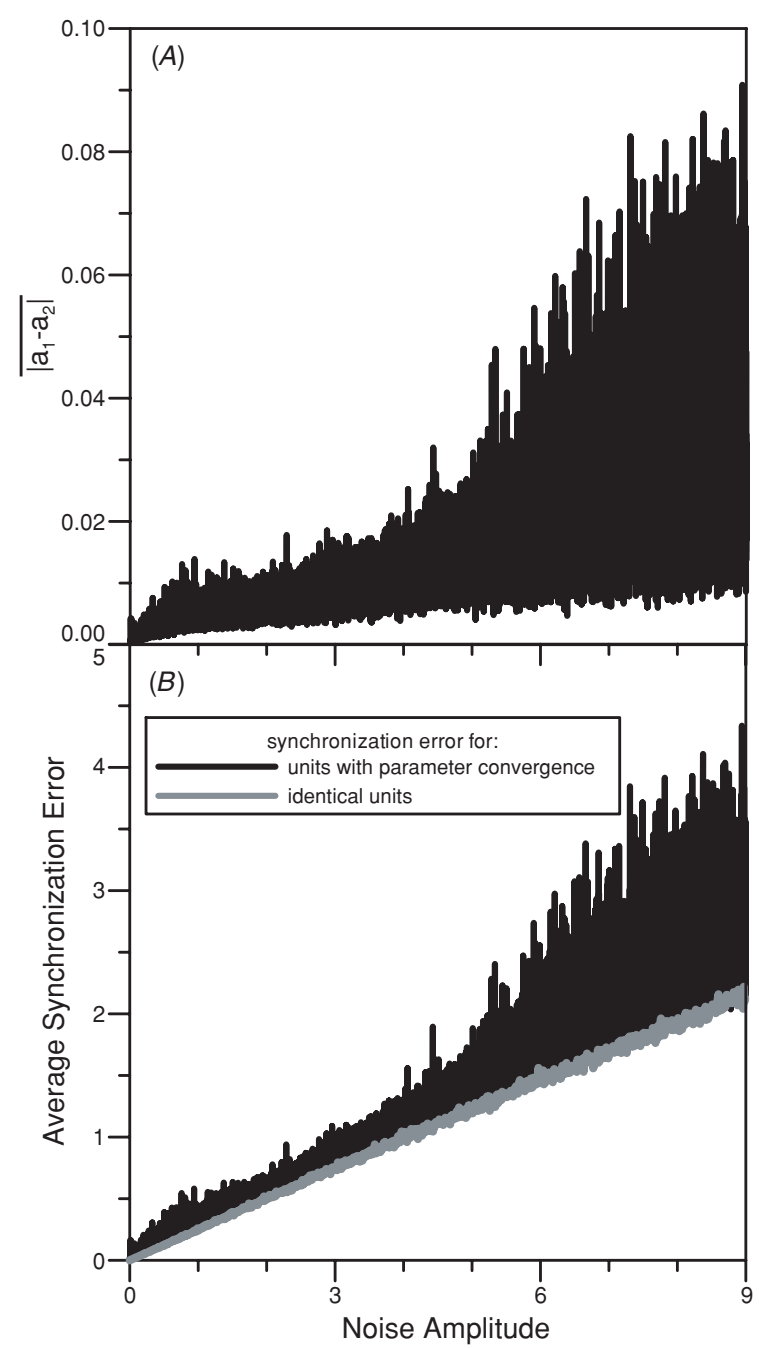

Figure 12. Synchronization and adaptation quality as a function of the noise amplitude for coupled Rössler oscillators. (A) Plot of the convergence of the control parameters; $(B)$ the synchronization error. The black line denotes the synchronization error of the two adapting units $\left(a_{1}=0.15\right.$ and $\left.a_{2}(0)=0.25\right)$. The grey curve denotes the synchronization error of two identical oscillators $\left(a_{1}=a_{2}=0.15\right)$ in the presence of noise.

In order to study the convergence of the two coupled oscillators in the presence of noise, we added a term $A_{N} \xi_{1,2}(t)$ to the $y$-coordinate of the control and target systems, respectively; $A_{N} \in[0,10]$ and $\xi(t) \in(-1,1)$ is a random variable obtained from a uniform distribution. The convergence error for both the control parameters and synchronization are plotted in figures $12(A)$ and $(B)$, respectively. Additionally, in figure 12(B), we plotted (grey line) the synchronization error for two identical Rössler oscillators (both $a_{1}=a_{2}=0.15$ throughout the simulation). As could be expected, the quality of convergence is notably reduced in the presence of noise. The synchronization error, as well as the parameter convergence error, is stable for $A_{N} \leqslant 3.0$. Above this value both errors increase sharply. This is due to the fact that the distributions used to calculate the entropy of the control and target become significantly perturbed by the noise. 


\section{Conclusions}

In summary, we used entropy estimation to quantify the dynamical properties of the coupled system and used its difference to control the adaptation and synchronization of two coupled systems. The adaptation is achieved via internal modifications of the control parameter of the target. The entropy is estimated from evolving distributions that are constantly updated throughout the dynamics and reflect the changes in the properties of dynamical trajectories of the two systems.

The method does not incorporate any a priori information about the control system and is solely based on the distribution of the events characterizing the dynamical trajectories of the coupled system. The implementation and properties of the proposed method are shown on a system of two coupled logistic maps and also on two coupled Rössler oscillators. In both cases the difference in entropies of the distributions of one variable (i.e., the distributions of the $x$-coordinate of the crossing of the specified Poincaré section for the Rössler oscillator and the distributions of the iterate values for the logistic maps) controls the changes in the single control parameter of the target system. It may be possible to generalize this onedimensional adaptation process to multiple dimensions. This could be done by separating the time scales of convergence of the different control parameters. The convergence of the faster changing control parameter would minimize the $\left|S_{1}-S_{2}\right|$ for the value of the slower changing control parameter. The search would continue until the $S_{1}-S_{2}=0$. Another way to control multiple control parameters would be to define independent distributions that would separately determine the respective changes.

The fact that the convergence of the target parameter is effectively established through the statistical properties of the intersection of its trajectory with the Poincare section allows for experimental application of the control scheme. One can use experimentally devised Poincaré sections [26] to control the trajectories of coupled elements having different dynamical trajectories. Moreover, we have shown earlier [27] that such crossings provide enough information to synchronize two otherwise independent units.

The lack of requirement of any knowledge about dynamical equations of the coupled systems could allow the presented method to mimic the adaptive processes in nature, specifically in biological systems, where neural spike timings for example, could be used to control the neuronal adaptation processes. Thus, the neuron would be (at least theoretically) able to adjust its control parameters (specific membrane conductances) to match its activity to the properties of the incoming signal from other neurons and synchronize with them forming functional units during information processing. The relative robustness of the presented adaptation scheme to noise indicates that the patterns of activity of the neurons could converge to the desired trajectory even in the case when significant noise is present in the system; the impossibility of which was thought to be one of the major obstacles for neurons to use the timing-dependent codes [28].

\section{References}

[1] Parlitz U, Kocarev L, Stojanovski T and Preckel H 1996 Encoding messages using chaotic synchronization Phys. Rev. E 534351

[2] Rulkov N F, Suschik M M, Tsimring L S and Abarbanel H D I 1995 Generalized synchronization of chaos in directionally coupled chaotic systems Phys. Rev. E 51980

[3] Rosenblum M G, Pikovsky A S and Kurths J 1997 From phase to lag synchronization in coupled chaotic oscillators Phys. Rev. Lett. 784193

[4] Pecora L M and Carroll T L 1990 Synchronization in chaotic systems Phys. Rev. Lett. 64821 
[5] Meyer-Lindenberg A, Ziemann U, Hajak G, Cohen L and Berman K F 2002 Transitions between dynamical states of differing stability in the human brain Porc. Natl Acad. Sci. USA 9910948

[6] Luthi A and McCormick D A 1998 H-current: properties of a neuronal and network peacemaker Neuron 21 9-12

[7] Sauvé K 1999 Gamma-band synchronous oscillations: recent evidence regarding their functional significance Conscious. Cogn. 8 213-24

[8] Basa̧r E, Baş̧r-Eroğlu C, Karakaş S and Schürmann M 1999 Are cognitive processes manifested in event-related gamma, theta and delta oscillations in the eeg? Neurosci. Lett. 259 165-8

[9] Singer W 1999 Neuronal synchrony: a versatile code for the definition of relations? Neuron 24 49-65

[10] Munk M H J, Roelfsema P R, König P, Engel A K and Singer W 1996 Role of reticular activation in the modulation of intracortical synchronization Science 272 271-4

[11] Klimesch W 1999 Eeg alpha and theta oscillations reflect cognitive and memory performance: a review and analysis Brain Res. Rev. 29 165-95

[12] Gray C M and Singer W 1989 Proc. Natl Acad. Sci. USA 861698

[13] Roelfesma P R, Engel A K, König P and Singer W 1997 Visuomotor integration is associated with zero time-lag synchronization among cortical areas Nature 385 157-61

[14] Lam Y, Cohen L B, Wachowiak M and Zochowski M 2000 Odors elicit three different oscillations in the turtle olfactory bulb J. Neurosci. 20 749-62

[15] Laurent G 1996 Dynamical representation of odors by oscillating and evolving neural assemblies Trends Neurosci. 19 489-96

[16] deCharms R and Merzenich M 1996 Primary cortical representations of sounds by coordination of action potential timming Nature $\mathbf{8 1} 610-3$

[17] Engel A K, König P and Singer W 1991 Direct physiological evidence for scene segmentation by temporal coding Proc. Natl Acad. Sci. USA 88 9136-40

[18] von der Malsburg C 1994 The correlation theory of brain function Models of Neural Betworks II (Berlin: Springer)

[19] von der Malsburg C 1985 Nervous structures with dynamical links Ber. Bunsenges. Phys. Chem. 89 $703-10$

[20] Schurmann T and Grassberger P 1996 Entropy estimation of symbol sequences Chaos 6414

[21] Schouten J C, Takens F and van den Bleek C M 1994 Maximum-likelihood estimation of the entropy of the attractor Phys. Rev. E 49126

[22] Macek W M and Redaelli S 2000 Estimation of the entropy of the solar wind flow Phys. Rev. E 626496

[23] Bezerianos A, Tong S and Thakor N 2003 Time-dependent entropy estimation of eeg rhythm changes following brain ischemia Ann. Biomed. Eng. 31221

[24] Shannon C E 1949 The Mathematical Theory of Communication (Chicago IL: University of Illinois Press)

[25] Astakhov V, Hasker M, Kapitaniak T, Shabunin A and Anishchenko V 1998 Effect of parameter mismatch on the mechanism of chaos synchronization loss in coupled system Phys. Rev. E 585620

[26] Fountain G O, Khakhar D V and Ottino J M 1998 Visualization of three dimensional chaos Science 281683

[27] Zochowski M 2000 Intermittent dynamical control Physica D 145181

[28] Shadlen M N and Movshon J A 1999 Synchrony unbound: a critical evaluation of the temporal binding hypothesis Neuron $\mathbf{2 4} 67-77$ 